\title{
Detection of Regional Cardiac Wall Motion Abnormalities in Myocardial Infarction by Cardiac Wall Kymography
}

\author{
Kohji Tamura, M.D., Nobuo Furukawa, M.D., Yutaka Arai, M.D., \\ Hiroshi Murooka, M.D.,* Yoshiaki Sarto, M.D.,** \\ and Masahiko OKaDA, M.D.***
}

\begin{abstract}
Summary
The new system of cardiac wall kymography was proposed based on the results with our modified device. The cardiac wall motion kymography taken from a portion of the precordial area was examined for its ability to detect wall motion abnormalities. Furthermore, the 3 dimensional graphic display of the cardiac wall kymogram map was attempted by computer. This new approach looks promising because of the enhanced ability to recognize cardiac wall motion abnormalities in myocardial infarction.
\end{abstract}

Additional Indexing Words:

Three-dimensional cardiokymography Computer analysis Systolic bulge Non-invasive method

$\mathrm{N}$ order to recognize the occurence of myocardial ischemia the detection and the estimation of the regional cardiac wall motion abnormalities are necessary rather than the examination of the global ventricular dysfunction. ${ }^{11}$ The left ventricular angiography has been invasively the standard method to approach to this clinical diagnosis. Obviously, however, there are certain limitations in this method to assess the cardiac wall motion abnormalities. Therefore, a non-invasive method is expected to be developed to answer this clinical need." The non-invasive techniques, such as apex cardiography, carotid pulse tracings, phonocardiography, radioisotopic methods, echocardiography, and others, were introduced and certainly gave us informations that were useful clinically. ${ }^{2,3)}$ However, these methods do not satisfactorily answer to our clinical needs in its accuracy, precision, convenience, and

From the First Department of Medicine, Niigata University School of Medicine, * Central Diagnostic laboratory, Niigata University Hospital, ** Faculty of Engneering, Niigata University, and *** Department of Neurophysiology, Brain Research Institute, Niigata University School of Medicine, Niigata.

Address for reprint: Kohji Tamura, M.D., the First Department of Medicine, Niigata University School of Medicine, Niigata-shi 951, Japan.

Received for publication December 6, 1979. 
others. ${ }^{2), 31}$

The cardiokymography (CKG) was newly developed by Vas et al in 1967 in order to recognize the cardiac wall motion abnormalities non-invasively." This method uses a simple electromagnetic displacement transducer to sense the tissue movement beneath it. This method has been well accepted as the useful non-invasive method to detect the cardiac wall motion abnormalities mainly because of its low cost, portability and ease of application. ${ }^{1,4)-12)}$ Unfortunately, however, this device of cardiokymography is not commercially available yet. Practically this method is limited by low supply of these devices by Vas. Therefore, we modified and developed the device of cardiokymography by our own hands based on the principle proposed by Vas et al. ${ }^{13}$ ) Thus we could obtain the better stability than his original model of the device and could use this divice clinically already, ${ }^{13), 14}$ )

The objects of this study were to examine our new modified system of cardiac wall kymography (CKG) regarding the ability to detect the cardiac wall motion abnormalities in myocardial infarction with the records (i) at rest, (ii) during exercise, and (iii) with the entirely newly developed 3 dimensional graphic display computer analysis of the cardiac wall motion by taking the precordial CKG maps.

\section{Methods and Data Analysis}

\section{The system of CKG (Fig. 1)}

The system of CKG in our hand is shown in Fig. 1. A electromagnetic displacement transducer was made of the flat coil of $6 \mathrm{~cm}$ in diameter as a part of a high frequency low power oscillator. ${ }^{41-12}$ This transducer was placed on the precordial chest wall and was fixed by a band to avoid the induction of the noise by unexpected movement of the transducer itself. The change of the distance between the coil and the tissue caused the change of the inductance of the circuit made by coil and condenser. ${ }^{12)}$ Thus, this change in frequency was converted into a voltage proportional to the tissue motion. ${ }^{12}$ ) CKG records the tissue movement under the transducer. Therefore, the moved tissue that was detected by CKG could be either the chest wall or cardiac epicardial wall, or both of them. Since CKG utilizes both magnetic field and electrical field to detect the tissue movement, the detected moving tissue could be mainly the chest wall or the cardiac epicardial wall. The dominance of either tissue would be determined by (1) the distance between the tissue and the coil since cardiokymographic output decreases exponentially with distance and (2) the magnitude of the movement of either tissue comparatively. ${ }^{12), 13}$ ) Our newly developed CKG device was connected with the recorder (Photocorder, Yokogawa Co).

2. CKG from a portion (Fig. 2)

CKG from a portion of the precordial area was taken simultaneously with electrocardiogram, phonocardiogram, and carotid arteriogram so that we could analyse the tracing, correlating this with cardiac cyclic events as shown in Fig. 2. 


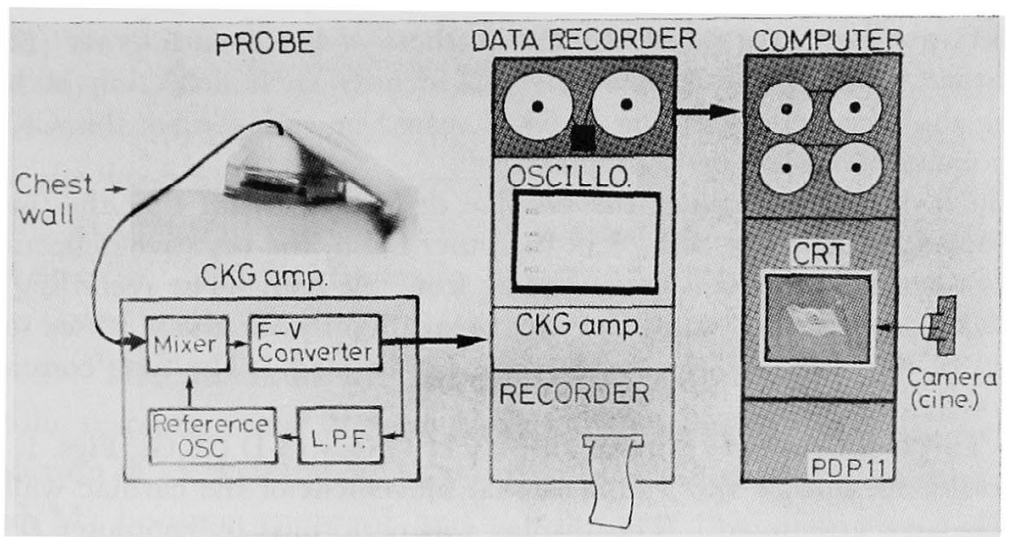

Fig. 1. System of cardiac wall kymography. CKG from a portion of the precordial area or 3 dimensional graphic display of CKGs obtained from CKG map of the precordial area are shown. $F-V=$ frequency-voltage; OSC $=$ oscilloscope; L.P.F. = low pass filter $;$ amp. =amplifier $; \mathrm{CRT}=$ cathode ray tube; OSCILLO $=$ oscilloscope.
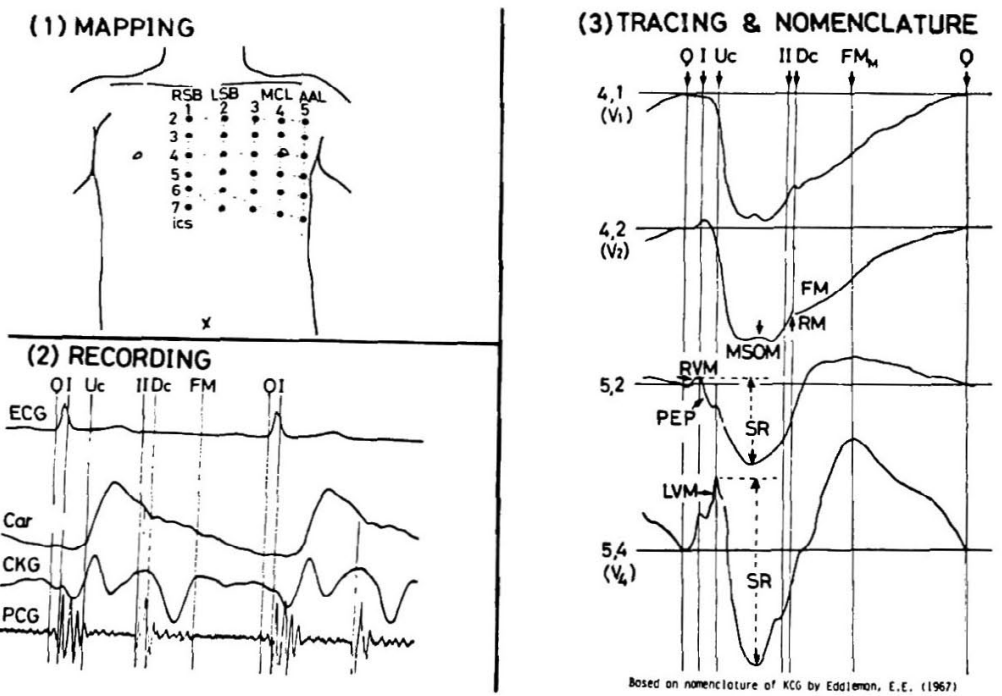

Fig. 2. CKG taken from a portion of the precordial area. Mapping of CKG was performed. The recording of each of CKG was performed simultaneously with electrocardiogram, carotid arteriogram (Car), and phonocardiogram (PCG). The nomenclature of each of the deflections in CKG was made referring to that of kinetocardiogram proposed by Eddleman. $Q=Q$ wave of ECG; I = 1st heart sound; $\mathrm{Uc}=$ upstroke time in carotid arteriogram; $\mathrm{II}=$ second heart sound; $\mathrm{Dc}=$ dicrotic notch in carotid arteriogram; $\mathrm{FM}_{\mathrm{M}}=$ maximal filling movement; $\mathrm{FM}=$ filling movement; $\mathrm{RM}=$ rapid movement; $\mathrm{MSOM}=$ mid-systolic outward movement; $\mathrm{RVM}=$ right ventircular movement; $\mathrm{PEP}=$ pre-ejection period; $\mathrm{SR}=$ systolic retraction; $\mathrm{LVM}=$ left ventricualr movement; $\mathrm{RSB}=$ right sternal border; $\mathrm{LSB}=$ left sternal border; $\mathrm{MCL}=$ mid-clavicular line; $\mathrm{AAL}=$ anterior axillary line. 
Since CKG tracings were mostly similar to those of kinetocardiogram (KCG), the nomenclature proposed by Eddleman ${ }^{15)}$ to identify each deflection of KCG was utilized so that the understanding of CKG would be easier, since this CKG is new and no nomenclature has been proposed yet.

In each of the deflections, the positive deflection meant that the tissue under the coil moved close to the coil. On the other hand, the negative deflection meant that the tissue under the coil moved away from the coil. The recording was performed during expiration by holding the breath lightly for about $10 \mathrm{sec}$ so that the movement of the chest wall by respiration was avoided. The time constant of the system was $1.5 \mathrm{sec}$.

3. Three-dimensional graphic display of CKGs (3-D GKGs, Figs. 1, 2, and 5)

In order to analyse the 3 dimensional movement of the cardiac wall in time, 3-D CKGs were attempted. This display was performed by computer (PDP 11 or 12, DEC Co, USA) and was displayed on CRT of this computer (Fig. 1). The precordial CKG map was performed by recording CKG at the multiple portions of the precordial area. $\mathrm{CKG}$ was taken sequentially starting from $(2,1)$, namely at the crossing point of the 2nd intercostal space and the right sternal boarder (R.SB), ending at $(7,5)$, which was at the crossing point of the 7 th intercostal space and the anterior axillary line. By this mapping technique, altogether 30 records were obtained and recorded on magnetic tape (Fig. 1 and 2). Data processing by computer for 3-D CKGs were performed in a manner which gave a $3 \mathrm{D}$ analysis of the electrocardiogram's graphic display which was recently developed by one of the authors. ${ }^{16)}$ The imput of CKG into the computer was performed by selecting each of one beat of CKG displayed on the CRT. For practical purposes, the zero reference point was taken at the beginning of a $q$ wave of limb lead ECG taken simultaneously. This ECG and CKG were simultaneously digitized by analog-to-digital converters and were stored in the core memories. This visual selection of CKG on $\mathrm{CRT}$ was repeated 30 times. The total time consumed for this selection was about $20 \mathrm{~min}$.

The production of 3-D CKGs was performed as follows. ${ }^{16}$ In computer memories, thirty digitized CKGs were arranged in a synchronous time base. With the first point of all $30 \mathrm{CKGs}$, the first 3-D CKG display was produced. This procedure was repeated until the last point of all CKGs were processed. The movement of the tissue close to the coil was shown as the positive deflection on the vertical axis as before. The parallel 16 lines of the precordial area wree drawn along the horizontal axis of the graph in order to give a cubic effect. The total computation time for one graph of CKG was about $2.5 \mathrm{sec}$. In order to show the dynamic movement of the graph the animation display of 3-D CKGs was made. Namely every computed graph was once stored and displayed on CRT sequentially. Finally this animation was taken by either camera or cinecamera for the analysis of the dynamic events of the cardiac wall motion.

\section{Results}

1. CKG from a portion on the precordial area

a) CKG in a healthy person (Fig. 2)

CKG tracings of a healthy person are shown in Fig. 2. At the portion 
of $(4,1)$, namely at $V_{1}$ position of electrocardiogram, CKG showed only large negative deflection starting from $q$ in ECG until the end of the diastole. The mid-systolic outward movement (MSOM) and the rapid movement (RM) of the ventricles were recognized. At the point of $(4,2)$, namely $V_{2}$ position of ECG, small positive deflection appeared, reflecting the movement of the ventricle. The mid-systolic outward movement became more apparent. At the portion of $(5,2)$, the inital right ventricular movement was recognized. The notch in the pre-ejection period (PEP) was also visible. The systolic retraction and the positive filling movement were also recognized. In the portion of $(5,4)$, namely $\mathrm{V}_{4}$ position of $\mathrm{ECG}$, the left ventricular movement (LVM) became apparent and the peak of this deflection was slightly delayed compared with that of the right ventricular movement. The systolic retraction and the subsequent filling movement became more apparent.

Thus, we were able to obtain this $\mathrm{CKG}$ tracing at any point on all the patients studied so far in more than 150 cases.

b) CKG in myocardial infarction (Figs. 3 and 4)

In a case of myocardial infarction (48 y.o., male with old anterolateral myocardial infarction with definite left ventricular aneurysm), CKG which was taken at $(4,4)$, namely at $V_{4}$ point of ECG, is shown in Fig. 3. Compared with the normal tracing that is shown as a dotted line in this figure, the reversed positive deflection was noted during the systole and again the inverted deflection was observed during the diastole. Therefore, this paradoxical movement of the cardiac wall would be correlated with left ventricular aneurysm formed after myocardial infarction.

Furthermore, the ability to detect wall motion abnormalities during the induced myocardial ischemia by CKG was examined. In a case of myocardial infarction with definite left ventricular dyskinesia exercise induced an anginal attack. CKG (Fig. 4) at the rest stage of this patient was noted to already have a late systolic bulge. After the anginal attack, ST segment was de-

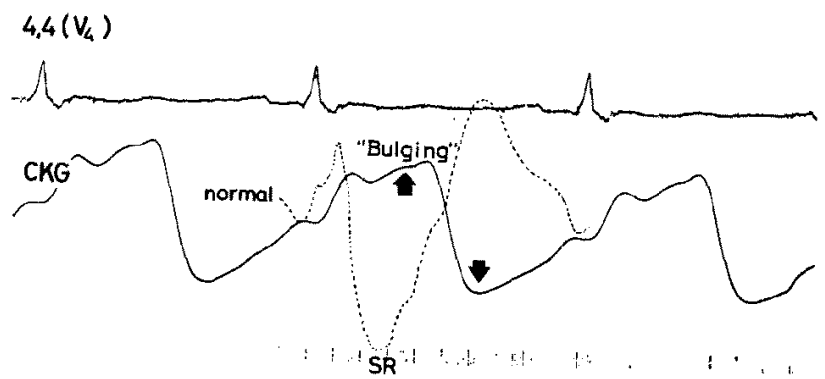

Fig. 3. CKG in myocardial infarction with left ventricular aneurysm. The indicating arrows show the paradoxical motions (solid line) compared with normal CKG tracing shown as a dotted line. 


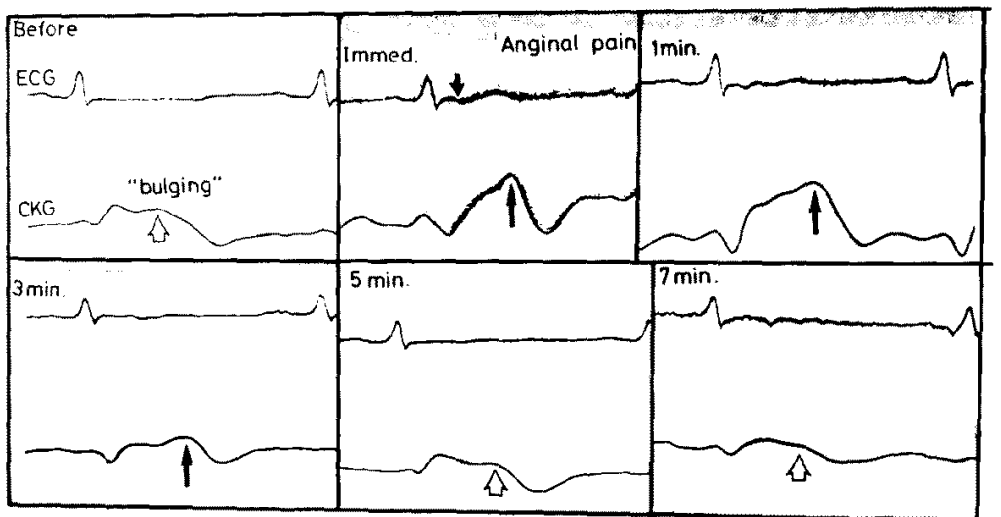

Fig. 4. CKG taken during induced anginal pain in myocardial infarction. CKG showed the "bulge", before the accurance of pain. Immediately after the occurrence of the anginal pain (dark bar) ST segment depression was noted and the late systolic bulge became more apparent (arrows).

pressed. Simultaneously enhancement of the systolic bulging movement of the cardiac wall during late systole was definitely observed. This CKG change was observed before appearance of anginal pain and the electrocardiographic abnormality. Therefore, CKG was definitely sensitive enough to detect myocardial ischemia in this case.

2. Three dimensional graphic display of cardiac wall kymogram (3DCKGs, Figs. 5, 6, and 7)

a) 3-D CKGs in a healthy person

Our approach using 3-D graphs to display CKG gave us a clear picture and made an analysis of cardiac wall motion as a whole possible. The bird's eye view of the anterior cardiac wall motion of a healthy person is shown in Fig. 5. At first a CKG map was taken and displayed on a single plane. This plane was viewed from 30 degrees to the right and 45 degrees to the footward direction. At the right upper quadrant of Fig. 5, the presumable anatomic cardiac wall scheme was drawn. During systole, as indicated by the terminal point of the electrocardiogram at the right shoulder of each graph, the pulmonary arterial wall distended, the right ventricular wall retracted and the interventricular septal area protruded. During diastole, the motion of each portion of the cardiac wall seemed to be reversed. Namely, the pulmonary arterial wall retracted, the right ventricular wall protruded and the interventricular septal area retracted. These changes in the wall motion seemed reasonably correlated with the presumably physiological cardiac wall motion itself.

The detail of this normal cardiac wall motion in a healthy 43 y.o. male 


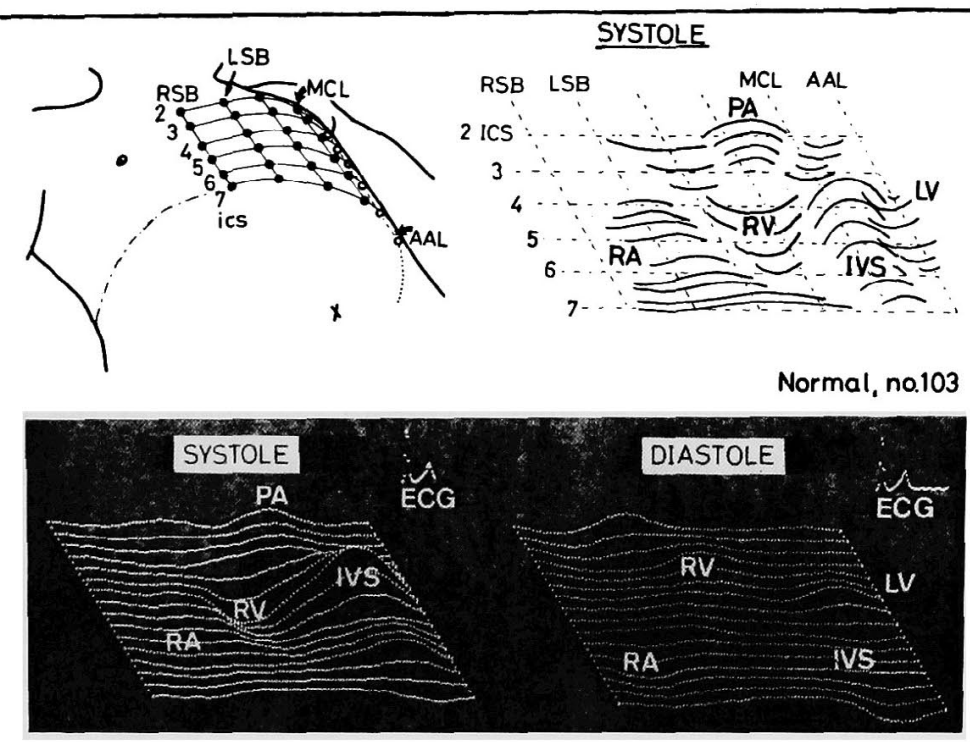

Fig. 5. Three dimensional graphic display of CKGs in a healthy person. At the right upper quadrant, the presumptive schematic anatomical portion of the cardiac wall by CKG was drawn. The graphs of both systole and diastole are shown.

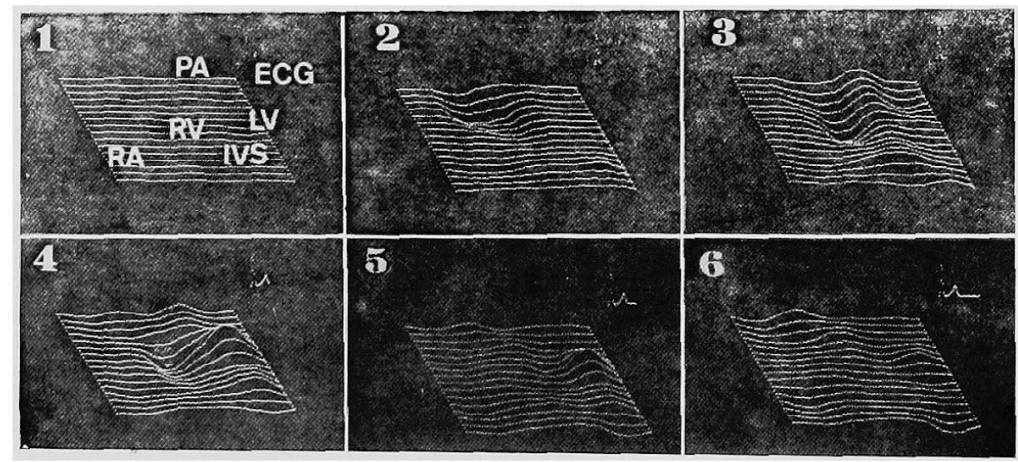

Fig. 6. Three dimensional graphic display of GKGs in a healthy person, 43 y.o. male. The numbers on 3-D CKGs show the sequence of recording after beginning of ventricular activation.

is shown in Fig. 6. The retraction, presumably of the right ventricular wall, started in the right upper corner, at the beginning of the isovolumic contraction period (phase 1) and the elevation of the lower part of the right ventricular wall was also noted (phase 2). At phase 3, the retraction of the upper part of the right ventricle, and the protrusion of the lower part of it, became more apparent toward the end of the isovolumic retraction period. At the beginning of the ejection period, the whole right ventricle retracted and the interventricular portion started to protrude. During diastole, the pulmonary 
arterial wall retracted, the right ventricle distended and the interventricular septum retracted. This movement became more apparent at the terminal of diastole (phase 6). At this time, even the right atrium seemed to be distended.

b) 3-D GKGs in myocardial infarction with left ventricular aneurysm (Fig. 7)

In a case of myocardial infarction (48 y.o., male with anterior wall infarction with angiographically proved left ventricular dyskinesia) 3-D CKGs were examined in a similar manner. During the phase of the isovolumic contraction period, the prominent protrusion of the lower right portion of this display was already noted. At the end of this period (phase 3), this protrusion became more prominent and the right ventricular retraction was noted only at the upper part of the right ventricle (phase 3). At the beginning of the ejection period, this movement was more prominent (phase 4). During ejection, the right lower area of the graph became more protruded even though the right ventricular retraction occured only at the upper part (phase 5). During diastole, the above mentioned movements were completely reversed. Namely, the prtruded wall was apparently retracted and, the retracted right ventricle was distended during this period. Therefore this area might be thought to be the paradoxical motion area, namely "bulging area ", and this would correspond to the ventricular wall dyskinetic area formed after myocardial infarction.
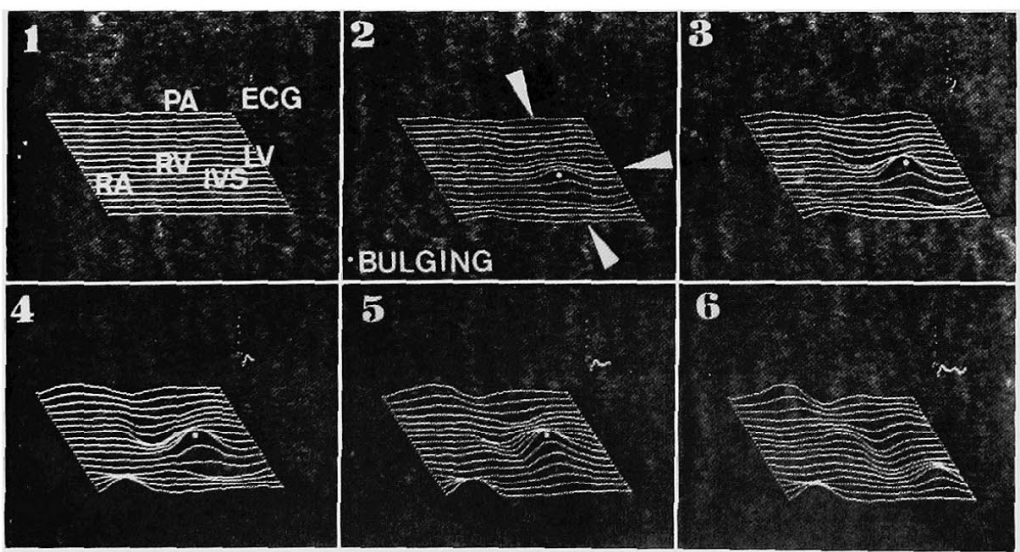

Fig. 7. Three dimensional graphic display of CKGs in a myocardial infarction of a 48 y.o. male with definite left ventricular aneurysm. The numbers indicate the sequence of the displayed graphs taken after the beginning of the ventricular activation. 


\section{Discussion}

There is no question about necessity to recognize the changes of the cardiac wall motion for detection of coronary heart disease. The importance of recognition of this disorder in coronary heart disease is that the wall motion abnormality precedes the electrical excitation in myocardial ischemia and it facilitates recognition of magnitude and extent of myocardial ischemia. ${ }^{1}$ Therefore, various non-invasive diagnostic methods for the recognition of cardial wall motion abnormalities have been investigated. ${ }^{2)}$ Precordial vibration recording techniques, ${ }^{2)}$ such as kinetocardiography (Eddleman, Harrison), vibro-cardiography (Agress), radionuclide scanning, echocardiography, roentgen kymography, electrokymography, radar kymography, and others have been developed. ${ }^{3}$ )

Cardiokymography is new and still limited to only special research groups in the world, because of the limit of supply of the apparatus itself. ${ }^{4-12}$ ) However, the significance of recognition of cardiac wall motion abnormalities has become well accepted throughout the world, even though there are many problems still to be solved before this method can be introduced in the practical clinical field, since even the naming to this method is not established yet. ${ }^{4-12}$ ) This method has been referred to by various names, such as: displacement cardiography, ${ }^{6), 7)}$ displacement apex cardiography, ${ }^{11}$ focal displacement cardiography, ${ }^{8)}$ cardiokymography, ${ }^{10)}$ and we chose the more specific term "cardiac-wall kymography". This change in nomenclature is a result of this new approach concerning the evaluation of the method itself as will be discussed later.

1. Criticism against the method

In CKG, the electromagnetic field is applied to the chest wall, thereby measuring the 2 sources of output simultaneously. ${ }^{6}$ One of the sources is the air gap distance between the tissue and the probe, and the other is the movements occuring within the tissue itself. Therefore, the dominant source of the signal is constantly changing. Its dominancy is determined by: a) distance of probe either from the chest wall and the cardiac wall, since CKG output is exponential with distance, and b) magnitude and velocity of the movement of either the chest wall or the cardiac wall." However, the basic principle that $\mathrm{CKG}$ tracing represents the true analog wave of the cardiac wall motion itself is well accepted today. ${ }^{9}$ (Therefore, we named this CKG cardiac-wall kymography rather than call it cardiokymography.

The benefits of this method are stressed because of its simplicity, relatively low cost of the divice and the ease with which cardiac wall motion can be measured. However, according Vas' data the device lacked stability. There- 
fore, we modified his original design of the device ${ }^{13), 14)}$ and this cnablcd us to obtain more stable tracing characteristics. By arriving at this degree of stability, it then became possible to obtain the map of CKG and display it in the 3-dimensional graphs by computer.

Critics on this method had pointed to 3 basic flaws, a) CKG records only a vectorial component of the cardiac wall movement that is perpendicular to the coil,$^{91}$ b) its records are usually not obtainable at all precordial areas by Vas' apparatus, ${ }^{9}$ " and c) there is no calibration in CKG so far. ") In order to solve these problems, we first developed better stability in the apparatus. It then became possible to record CKG at all precordial areas in every one of more than 150 patients studied so far. Therefore, the problem concerning recordability was solved.

2. Tracing from a portion of precordial area

The tracings obtained by CKG were very similar to these obtained by KCG. ${ }^{15}$ For convenience sake, the nomenclature proposed by Eddleman was temporarilly applied to $\mathrm{CKG}$, since no attempts to name CKG tracings had been made. ${ }^{15)} \mathrm{KCG}$ itself is not so popular even today, since there are certain limitations to this method such as a) poor stability, b) the system complexity as a clinical device, and c) difficulties in differentiating between normals and abnormals. In CKG the former 2 problems in $\mathrm{KCG}$ were already solved. Therefore, we tried to solve the third problem in KCG. In an attempt to overcome this problem, 3 dimensional graphic display of CKGs were taken as will be discussed later.

The abnormal motions in CKG were found in each of the cardiac cycles. The systolic outward movement of the cardiac wall which was the reverse of the normal movement, was definitely noted. This abnormal movement was shown to represent real dyskinesia of the wall in a dog experiment in which a directly implanted length gauges or ultrasonic crystal was applied in acute myocardial ischemia." Moreover, this paradoxical motion of the cardiac wall was similarly seen in both CKG and left ventriculography in coronary heart disease. ${ }^{9 !}$

On the physical stress test, CKG was also shown to be useful as an adjunct to electrocardiography. Namely, CKG showed a false positive of $6 \%$ in patients without angiographically documented coronary heart disease and true positive of $76 \%$ in CKG with angiographically documented coronary heart disease. ${ }^{10)}$ We could confirm the occurence of changes in the tracing as shown.

3. Three dimensional graphic display of CKGs

As described previously, the difficulties in differenciating the abnormal from the normal pattern were one of the weak points of KGG.15),17) This 
difficulty should still be solved in CKG, since CKG tracing from a portion of the precordial area is very sensitive to the location and the variability between individuals is great. This weak point may be solved by reducing the size of the probe. However, some limitations of CKG will always exist. Therefore, we attempted to solve this problem by making the 3-dimensional graphic display based on the CKG precordial map. The procedure for the data analysis was already published. ${ }^{16)}$

The CKG graph obtained showed the subtle motion of the cardiac wall and we succeeded in analysing the timing, and the extent of the diseased cardiac wall. In a healthy person, the movement of the cardiac wall was smooth and tremendous movement of the wall was noted. This movement was presumed to be normal based on the physiological background, even though there had been no similar display of the cardiac wall motion as a whole. Therefore, this approach enabled us to measure the timing, extent and magnitude of the diseased cardiac wall. But, much more work remains to be done before any definite conclusions can be drawn.

In order to test this approach in myocardial infarction, a CKG graph was made. The expected paradoxical motion of the cardiac wall was definitely seen giving informations regarding location, extent and possibly magnitude. Therefore, this completely new approach would be beneficial in evaluating the cardiac wall to differentiate the normal from abnormal movements.

The effort to verify this idea is now being undertaken in our laboratory, since this approach has been proven to be fruitful in detecting cardiac wall motion abnormalities.

\section{References}

1. Swan HJC: Implications of regional cardiac mechanical function in myocardial ischemia and infarction-a strategy for presymptomatic detection. Very early recognition of coronary heart discase. International Congress Series 435, Excerpta Medica, p. 120, 1978

2. Dimond G, Ducnas A, Benchimol A: Apex cardiography. Am Heart J 72: 124, 1966

3. Tavel ME: Clinical Phonocardiography and External Pulse Recording. 2nd Ed, Year Book Medical Publishers, 1972

4. Vas R: Electronic device for physiological kinetic measurcments and detection of extraneous bodies. Ieee Transactions on Bio-Medical Engincering BME-14: 2, 1967

5. Vas R, Diamond GA, Wyatt HL, DaLuz PL, Swan HJC, Forrester JS: Noninvasive analysis of regional myocardial wall motion: cardiokymography. Am J Physiol 233: 700, 1977

6. Fenton TR, Vas R: Measuring characteristics of the displaccment cardiograph. Med Biologic Engineer 00: 552, 1973

7. Gay TC, Vas R, Pittman DE, Joyner CR: The displacement cardiograph. Circulation 53: 139, 1976

8. Valero A: Focal displacement cardiography for bedside detection of myocardial dyskinesis. Am J Cardiol 25: 443, 1970 
9. Diamond GA, Chag M, Vas R, Forrcster JS: Cardiokymography. Quantitative analysis of regional ischemic left ventricular dysfunction. Am J Cardiol 41: 1249, 1978

10. Pichler M, Vas R, Diamond D, Tziovini D, Silverberg R, Swan HJC, Forrester JS: Cardiokymographie. Nichtinvasive Beurteilung der regionalen myocardialen Wandbewegung zur Erkennung der koronalen Herzkrankheit. Z Kardiol 67: 695, 1978

11. Ollendorff F, Vash R, Valero A: Effect of exercise and acclimatization on displacement apex cardiogram in normal young subjects. Brit Heart J 33:37, 1971

12. Vas $R$, Joyner CR, Pitman DE, Gay TC: The displacement cardiograph. Ieee Transactions on Biomedical Engineering BME-23: 49, 1976

13. Saito $Y$, Ookubo Y, Tamura K, Arai Y: Modification and its characteristics of cardiokymography. Jap J Med Electr Biologic Engineer 16: 1, 1978 (abstr)

14. Arai $\mathrm{X}$, Tamura K, Murooka $\mathrm{H}$, Ozawa $\mathrm{T}$ : Mechanism of detection of ventricular wall motion by displacement cardiograph. Abstracts I of VIII World Congress of Cardiology, p 424, 1978 (abst)

15. Eddleman EE, Harrison TR: The kinetocardiogram in patients with ischemic heart disease. Progr Cardiovasc Dis VI: 189,1963

16. Okada M: 3-Dimensional graphic display of electrocardiogram. Jap Circulat J 40: 167, 1976

17. Strozzi $\mathrm{C}$, Cocco $\mathrm{G}$ : The kinetocardiogram during the isoproterenol test for the assessment of coronary heart disease. Cardiology 62: 277, 1977 